\title{
Left Atrial Appendage and Atrial Septal Occlusion in Elderly Patients with Atrial Septal Defect and Atrial Fibrillation
}

\author{
Ming Chern Leong ${ }^{1}$, Geetha Kandavello ${ }^{1}$, Azlan Hussin ${ }^{1}$, Deventhiran Permal ${ }^{1}$, and \\ Surinder Kaur Khelae ${ }^{1}$ \\ ${ }^{1}$ INSTITUT JANTUNG NEGARA
}

June 10, 2020

\begin{abstract}
Introduction: Elderly patients with ASD often present with chronic atrial fibrillation and large left to right shunt. This study reports the experience of left atrial appendage (LAA) and atrial septal defect (ASD) closure in patients with significant ASD and chronic atrial fibrillation. Methods / Results: We report six consecutive elderly patients with chronic atrial fibrillation and significant ASD who underwent LAA and fenestrated ASD closure from 1 January 2014 until 31 December 2019. All periprocedural and long term (>1 year) outcomes were reported. Six patients (Male: 33.3\%; Mean age: $66.8 \pm 3.3$ years) were included. Mean CHADS2, CHA2DS2-VASc $\neg$ and HAS-BLED scores were $2.33 \pm 0.82,3.83 \pm 0.75$ and $1.83 \pm 0.75$. Four patients underwent simultaneous procedure while 2 patients underwent a staged procedure. Procedural success was achieved in all patients. Total occlusion was achieved during LAA occlusion without device embolization prior to ASD closure. Patients who underwent simultaneous procedure had a shorter total hospital stay and lower total hospital stay. During a follow-up period of $32.8 \pm 19.4$ months, both the devices were well seated. No device-related thrombosis or erosion reported. All patients remained in atrial fibrillation. No patients experienced any thromboembolic stroke or transient ischemic attack. Conclusion: LAA and ASD closure is feasible and can be safely performed in the same seating in elderly patients with a significant atrial septal defect.
\end{abstract}

\section{ORIGINAL ARTICLE}

Left Atrial Appendage and Atrial Septal Occlusion in Elderly Patients with Atrial Septal Defect and Atrial Fibrillation

Ming Chern Leong MRCPCH ${ }^{\mathrm{a}}$, Geetha Kandavello $\mathrm{MRCP}^{\mathrm{a}}$, Azlan Husin $\mathrm{MRCP}^{\mathrm{b}}$, Deventhiren Perumal $\mathrm{BSc}^{\mathrm{c}}$, Surinder Kaur Khelae MRCP ${ }^{\mathrm{b}}$

1. Paediatric \& Congenital Heart Centre, Institute Jantung Negara (National Heart Institute), Kuala Lumpur, Malaysia.

2. Electrophysiology Unit, Department of Cardiology, Institut Jantung Negara (National Heart Institute), Kuala Lumpur, Malaysia.

3. Department of Imaging, Institut Jantung Negara (National Heart Institute), Kuala Lumpur, Malaysia.

4. Surinder Kaur Khelae, MRCP, Electrophysiology Unit, Department of Cardiology, Institut Jantung Negara (National Heart Institute), Kuala Lumpur, Malaysia.

Financial support : nil

Disclosure statement : No authors have any conflict of interest to disclose.

Brief Title : Atrial septal defect and chronic atrial fibrillation

Corresponding author:

Ming Chern Leong 
Paediatric \& Congenital Heart Centre

Institut Jantung Negara (National Heart Institute of Malaysia)

145, Jalan Tun Razak,

50400 Kuala Lumpur, Malaysia

Tel : +603-26178470; Fax : +603 - 2694 6478; Email : mcleong@ijn.com.my

OCID iD: 0000-0002-7891-8060

\section{Authorship :}

All authors declared to have contributed in the following manner:

1) Substantial contributions to the conception and design or the acquisition, analysis, or interpretation of the data.

2) Substantial contributions to the drafting of the articles or critical revision for important intellectual content.

3) Final approval of the version to be published.

4) Agreement to be accountable for all aspects of the work in ensuring that questions related to the accuracy or integrity of any part of the article are appropriately investigated and resolved.

\section{STRUCTURED ABSTRACT}

Introduction: Elderly patients with ASD often present with chronic atrial fibrillation and large left to right shunt. This study reports the experience of left atrial appendage (LAA) and atrial septal defect (ASD) closure in patients with significant ASD and chronic atrial fibrillation.

Methods / Results: We report six consecutive elderly patients with chronic atrial fibrillation and significant ASD who underwent LAA and fenestrated ASD closure from 1 January 2014 until 31 December 2019. All periprocedural and long term ( $>1$ year) outcomes were reported. Six patients (Male: 33.3\%; Mean age: $66.8 \pm 3.3$ years) were included. Mean $\mathrm{CHADS}_{2}, \mathrm{CHA}_{2} \mathrm{DS}_{2}-\mathrm{VAS}_{\mathrm{c}}$ and HAS-BLED scores were $2.33 \pm 0.82$, $3.83 \pm 0.75$ and $1.83 \pm 0.75$. Four patients underwent simultaneous procedure while 2 patients underwent a staged procedure. Procedural success was achieved in all patients. Total occlusion was achieved during LAA occlusion without device embolization prior to ASD closure. Patients who underwent simultaneous procedure had a shorter total hospital stay and lower total hospital stay. During a follow-up period of $32.8 \pm 19.4$ months, both the devices were well seated. No device-related thrombosis or erosion reported. All patients remained in atrial fibrillation. No patients experienced any thromboembolic stroke or transient ischemic attack.

Conclusion: LAA and ASD closure is feasible and can be safely performed in the same seating in elderly patients with a significant atrial septal defect.

\section{Word count (Abstract): 212 words}

\section{INTRODUCTION}

Left atrial appendage (LAA) closure is a well-established stroke prevention strategy as an alternative to systemic oral anticoagulant in selected patients with high-risk nonvalvular atrial fibrillation ${ }^{1,2}$. Its rationale lies in excluding the left atrial appendage where most clots form in the left atrium. Closure of the LAA is safe with a low, acceptable risk of complications ${ }^{1,2}$. Currently, LAA occlusion is extended to patients with high HAS-BLED score or those who cannot tolerate, refuse or contraindicated for long-term oral anticoagulation therapy $^{3}$. In all other patients, oral anticoagulant remains the preferred thromboprophylaxis in patients with atrial fibrillation.

Elderly patients with atrial septal defect (ASD) often present with heart failure, impaired exercise capacity and chronic atrial fibrillation. They are usually symptomatic and ASD closure is often indicated to alleviate 
symptoms. To our knowledge, there is no published guideline to guide management in this unique group of patients. Some centers may attempt rhythm control and ASD closure while others opt for rhythm control and ASD closure. We opted for off-label transcatheter closure of LAA and ASD, which treats both the large left to shunt while providing the patients with effective thromboprophylaxis. Both the procedures utilities similar equipment such as transesophageal echocardiogram and the approach, which places the long sheath into the left upper pulmonary vein during closure. Also, the presence of an ASD simplifies the process of the LAA closure. Both procedures may even be performed together. However, such an approach is unconventional and not well studied. The objective of this study was to describe the feasibility and safety of closing both the LAA and ASD in patients with ASD and chronic atrial fibrillation.

\section{METHODS}

This was a single-center, retrospective study. Between January 2014 and December 2019, all consecutive patients with ASD with chronic atrial fibrillation who were suitable for transcatheter occlusion were included in the study. Suitable subjects were defined as patients who had no significant coronary artery disease or cardiac pathology, which would otherwise obviate them from a transcatheter procedure. Patients with significant concomitant cardiac pathology may benefit from a cardiac surgery where all the pathologies can be treated in the same setting and hence, were excluded. All subjects underwent $\mathrm{CHADS}_{2}, \mathrm{CHA}_{2} \mathrm{DS}_{2}-\mathrm{VAS}_{\mathrm{c}}$ and HAS-BLED score assessment to evaluate the risk of thrombotic strokes and bleeding on Warfarin prior to the procedure ${ }^{2,4-6}$. Written consent was obtained before the procedures.

All procedures were performed under general anesthesia. A transesophageal echocardiogram was performed to assess the ASD for the adequacy of the interatrial septal rims, the normalcy of the pulmonary veins, the heart valves and the presence of the thrombi of the LAA. The LAA was profiled at $0^{\circ}, 45^{\circ}, 90^{\circ}$ and $135^{\circ}$, where the size, the length and the morphology of the LAA were assessed. Once deemed suitable, the femoral vessels were canulated. The LAA was occluded via conventional method using Watchman device. The details of the technique and device selection were as previously described ${ }^{6}$. After successful occlusion of the LAA, the subjects proceeded with the closure of the ASD. Saturation run was performed in routine fashion and the ASD occlusion was performed as previously described ${ }^{8}$. A 6 French pigtail catheter was positioned into the left ventricle to monitor the left ventricular end-diastolic pressure during the procedure. The ASDs were initially occluded with a sizing balloon while the left ventricular end-diastolic pressure is being monitored. A small fenestration was created onto the ASD occluder using the dilator of the long delivery sheath. Since the size of the delivery sheath corresponds to the size of the device, the size of the fenestration created was relative to the size of the atrial septal occluder (Figure 1). Post occlusion, the left ventricular end-diastolic pressure was measured to ensure that it did not exceed $20 \mathrm{mmHg}$ prior to releasing the septal occluder. All subjects had their ASD closed using Amplatzer or Amplatzer-like septal occluders.

In all patients, a transesophageal echocardiogram to assess the presence of residual flow around the devices, the formation of device-related thrombus and the stability of the atrial septal occluder was performed 45 days post procedure. If there was satisfactory occlusion of the LAA, which was defined as the presence of a residual flow of less than $5 \mathrm{~mm}$ around the device, the Warfarin was stopped and replaced by dual antiplatelet therapy until 6 months after the initial LAA occlusion. Thereafter, the subjects were kept on Aspirin indefinitely.

The subjects were followed up at an interval of 45 days, 3 months, 6 months and yearly. Long term follow-up for device-related complications and the presence of stroke or transient ischemic attack (TIA) was carried out during which, a transesophageal echocardiogram was performed where indicated. The fenestration at the atrial septal occluder was also evaluated.

\section{RESULTS}

\section{Baseline Characteristics}

A total of 18 patients above 60 years old underwent transcatheter ASD closure during this period and only 6 patients had chronic atrial fibrillation. The baseline characteristics of the six consecutive subjects are listed 
in Table 1. All patients had a right atrial area of more than $20 \mathrm{~cm}^{2}$ and expectedly, all were in chronic atrial fibrillation. The mean $\mathrm{CHADS}_{2}$ and $\mathrm{CHA}_{2} \mathrm{DS}_{2}-\mathrm{VAS}_{\mathrm{c}}$ scores were $2.33 \pm 0.82$ and $3.83 \pm 0.75$ while the mean HAS-BLED score was $1.83 \pm 0.75$. None of the subjects had a history of major bleeding and none were contraindicated to oral Warfarin. All these patients were symptomatic.

In the first subject, LAA was occluded first. Upon finding satisfactory occlusion of the LAA on transesophageal echocardiogram after 7 weeks, the ASD was closed. The initial concern was the possibility of incomplete closure of the LAA. Closure of the ASD with the metallic device would complicate further intervention to the LAA if the LAA closure was left with a significant residual flow or the device has embolized ${ }^{9,10}$. In the subsequent 4 subjects, the ASDs were closed simultaneously with the LAA. The LAA occluder was observed for five minutes for possible embolization prior to the ASD closure. In the last subject, ASD was performed in a live transmission to an interventional meeting; the LAA had to be performed 1 week before the live transmission. The patient was informed of the staged procedure to which she agreed. In that patient, fenestration was not created at the device, instead, a pre-manufactured fenestrated atrial septal occluder was used.

\section{Immediate Procedural Outcome}

Success LAA occlusion, defined as having a residual flow of $<5 \mathrm{~mm}$ around the device during the periprocedural transesophageal echocardiogram, was achieved in all patients. In patient 2, there was a small residual of $2 \mathrm{~mm}$ which was no longer seen in the follow-up transesophageal echocardiogram at 3 months.

All patient had only single interatrial communication and had large ASDs with significant left to right shunt. Three patients had pulmonary vascular resistance index more than 4 Woods $\mathrm{x} \mathrm{m}^{2}$. However, given the significant left to right shunting, the ASDs were closed. No patients required to have their ASD device fenestration enlarged to offload the left ventricle due to a significant increase in the left ventricular enddiastolic pressure, and expectedly, none had acute pulmonary edema immediately after the ASD closure. All patients were extubated successfully on table. All but two patients were discharged 1 day after the procedure. Subject 2 developed a mild femoral venous pseudoaneurysm after the ASD closure, which resolved with mechanical compression. Subject 3 was noted to have an esophageal injury after the procedure. Postprocedural CT scan revealed no air in the mediastinum and the injury resolved after 1 day. She was nursed in the hospital until she tolerated solid food orally. No device embolization noted. All patients demonstrated left to right shunting across the fenestrated atrial septal occlude immediately post closure. On further followup, the fenestration closed gradually, by 6 months follow-up, none of the fenestration flow persisted when interrogated via transesophageal echocardiogram. Subject 6 still had a fenestration flow at last follow-up, which was a little over 3 months after the ASD closure. Given more time, the fenestration is likely to close off spontaneously.

Patients who underwent ASD and LAA closure in the same setting (Group 1) were compared with patients 1 and 6 (Group 2) who underwent the procedures separately. The total duration and cost of the procedures were shorter in Group 1 compared to Group 2 (82 minutes vs. 136.5 minutes and USD 12,869.76 vs. USD 13,598.46). The average length of hospital stay in Group 1 was also shorter than Group 2 (4.5 days vs. 7 days). The total cost and hospital stay included those taken to treat the venous pseudoaneurysm and esophageal injuries, sustained by patients 2 and 3, who were both in Group 1.

\section{Long Term Follow-up}

The patients were followed up over a mean duration of $32.8 \pm 19.4$ months. All patients reported symptomatic improvement. There was an improvement in the NYHA functional class and exercise capacity. None of the patients reverted to sinus rhythm post-procedure. On transesophageal examinations, no device-related thrombosis was noted. Both the LAA and atrial septal occluders were in stable position with no erosion or migration of the devices. Repeated right heart cardiac catheterization was not performed as the echocardiogram did not suggest a significantly elevated pulmonary arterial pressure and these patients were asymptomatic. No stroke or TIA was reported in any of the subjects during the follow-up. No late mortality was observed. 


\section{DISCUSSION}

Transcatheter ASD closure is a well-established procedure. However, closure of ASD in the elderly population is to be performed with caution as many patients may have a masked restrictive left ventricular physiology, which is not detectable using routine echocardiographic parameters ${ }^{11}$. In the presence of a restrictive left ventricular physiology, more left atrial blood is shunted across the ASD to the right atrium and there is a decreased amount of blood flowing into the restrictive left ventricle, a physiology very much akin to a "Lutembacher's Syndrome" ${ }^{12}$. This often leads to symptoms of heart failure due to an increase in pulmonary blood flow and a decrease in cardiac output, which is a strong indication to close off the interatrial septal communication. Patients with old age, chronic obstructive airway diseases, diabetes mellitus, hypertension and ischemic heart disease are particularly at risk of developing restrictive left ventricular physiology ${ }^{13}$. Echocardiogram depicting severely dilated atriums should raise the suspicion of a restrictive left ventricular physiology even though conventional echocardiographic diastolic measurements do not suggest so. During balloon occlusion of the ASD, all pulmonary venous blood is forced into the left ventricle, causing an abrupt volume loading of the restrictive left ventricle leading to an increase in left ventricular end-diastolic pressure and acute pulmonary edema. Nevertheless, this does not preclude ASD closure. Previous attempt to condition the left ventricle by diuresing the patients $2-3$ weeks prior to the closure has yielded variable results. Creating a fenestration at the atrial septal occluder with as a pop-off to offload the left ventricle is generally a more accepted way of treatment ${ }^{13-14}$. Self-created fenestration usually closes off spontaneously. Its purpose is to buy time for the left ventricle to remodel while the fenestration closes gradually over time. To date, there is generally no consensus as the degree of elevation of left ventricular end-diastolic pressure that warrants the creation of fenestration or the size of the fenestration. However, our center has a low threshold of creating a small fenestration in patients with severely dilated atriums and the fenestration is deemed adequate if the left ventricular end-diastolic pressure post-closure is less than $20 \mathrm{mmHg}$.

Often, elderly patients with ASD also present with atrial fibrillation. Currently, there is no consensus as to how this group of patients should be treated. Generally, there is 2 mainstays of treatment - rhythm or rate control. One may attempt electrophysiological ablation to revert the patient to sinus rhythm prior to the closure of $\mathrm{ASD}^{16}$, or close the ASD and treat the atrial fibrillation medically. In the presence of atrial fibrillation combined with a $\mathrm{CHA}_{2} \mathrm{DS}_{2}-\mathrm{VAS}_{\mathrm{c}}$ score of more than 1 , there is a need for thromboprophylaxis ${ }^{5}$. Although anticoagulant therapy has been preferred choice for thromboembolic stroke, the increased risk of bleeding especially in the elderly population may be a factor for LAA closure. In addition, since atrial septal closure is indicated, there is a role in closing the left atrial appendage in the same setting. In the absence of an ASD, consideration is made to weigh the risks and benefits of LAA occlusion, especially procedural related complications such as the risk of cardiac tamponade and injury to the adjacent structures during trans-septal puncture. In patients with existing ASD, a trans-septal puncture is not necessary and hence the procedure is much simplified. Transcatheter ASD closure utilizes similar equipment, sheath and approach as LAA closure. The procedure time is slightly increased if both procedures are carried out together. Our series demonstrated a high rate of success in LAA closure. If the transesophageal echocardiogram immediately after the procedure shows total occlusion of the LAA, ASD closure can be performed right away. If there is any doubt to the LAA closure, there is always the option of deferring the ASD closure to a later date. Performing both procedures in the same setting brings about significant cost advantage and improved patient experience.

A recent report recommended performing these two procedures simultaneous in patients with enlarged atriums without atrial fibrillation ${ }^{17}$. We are of the opinion that many amongst those in sinus rhythm may continue to remain so for an extended period. Patients with paroxysmal or newly onset atrial fibrillation may be treated via transcatheter ablation. Indiscriminately closing the LAA during ASD closure increases procedural cost and may even increase the patient's risk for procedure-related complications ${ }^{17-18}$. We, therefore opined that such a simultaneous approach should be patient-specific rather than universal. In our case, we selected only patients with chronic atrial fibrillation. In this group of patients, LAA closure may be indicated even though patients do not have contraindications for oral anticoagulants. Our study offers the prospect of a novel indication for LAA closure in patients who are suitable candidates for transcatheter ASD 
closure with chronic atrial fibrillation. The presence of chronic atrial fibrillation can be an indication for left atrial appendage closure regardless of what the $\mathrm{CHADS}_{2}, \mathrm{CHA}_{2} \mathrm{DS}_{2}-\mathrm{VAS}_{\mathrm{c}}$ and HAS-BLED scores may be.

\section{CONCLUSION}

LAA closure, usually performed for patients with atrial fibrillation significant risk of thrombosis who cannot tolerated warfarin, can be performed safely and simultaneously in selected patients with significant ASD with chronic atrial fibrillation. Performing them together simplifies the procedure and may be performed regardless of the $\mathrm{CHADS}_{2}, \mathrm{CHA}_{2} \mathrm{DS}_{2}-\mathrm{VAS}_{\mathrm{c}}$ and HAS-BLED scores. This study may pave the way for a possible novel indication for left atrial appendage closure. However, larger scale study is indicated before such an indication can be widely recommended.

\section{ACKNOWLEDGEMENT}

Nil

\section{REFERENCE}

1. Boersma LVA, Schmidt B, Betts TR, et al. Implant success and safety of left atrial appendage closure with the WATCHMAN device: peri-procedural outcomes from the EWOLUTION registry. Eur Heart $J$. 2016;37(31):2465-2474.

2. Holmes DR, Doshi SK, Kar S, et al. Left Atrial Appendage Closure as an Alternative to Warfarin for Stroke Prevention in Atrial Fibrillation: A Patient-Level Meta-Analysis. J Am Coll Cardiol . 2015;65(24):2614-2623.

3. Kirchhof P, Benussi S, Kotecha D, et al. 2016 ESC Guidelines for the management of atrial fibrillation developed in collaboration with EACTS.Eur Heart J . 2016;37(38):2893-2962.

4. Gage BF, Yan Y, Milligan PE, et al. Clinical classification schemes for predicting hemorrhage: Results from the National Registry of Atrial Fibrillation (NRAF). Am Heart J . 2006;151(3):713-719.

5. Olesen JB, Lip GYH, Hansen ML, et al. Validation of risk stratification schemes for predicting stroke and thromboembolism in patients with atrial fibrillation: Nationwide cohort study. Bmj . 2011;342(7792):320.

6. Pisters R, Lane DA, Nieuwlaat R, et al. A novel user-friendly score (HAS-BLED) to assess 1-year risk of major bleeding in patients with atrial fibrillation: The euro heart survey. Chest . 2010;138(5):1093-1100.

7. Landmesser U, Holmes DR. Left atrial appendage closure: A percutaneous transcatheter approach for stroke prevention in atrial fibrillation. Eur Heart J . 2012;33(6):698-704.

8. Alwi M, Leong MC. Catheter-Based Interventions on Intracardiac Shunts .; 2014. 1127-1151p

9. Della Rocca DG, Horton RP, Di Biase L, et al. First Experience of Transcatheter Leak Occlusion With Detachable Coils Following Left Atrial Appendage Closure. JACC Cardiovasc Interv . 2020;13(3):306-319.

10. Pillai AM, Kanmanthareddy A, Earnest M, et al. Initial experience with post Lariat left atrial appendage leak closure with Amplatzer septal occluder device and repeat Lariat application. Hear Rhythm . 2014;11(11):1877-1883.

11. Ewert P, Berger F, Nagdyman N, et al. Masked left ventricular restriction in elderly patients with atrial septal defects: A contraindication for closure? Catheter Cardiovasc Interv . 2001;52(2):177-180.

12. King RL, Cleveland FM. Interauricular septal defect with mitral stenosis; the Lutembacher syndrome. Bull Mason Clin . 1949;3(1):13-20.

13. Gutierrez C, Blanchard DG. Diastolic heart failure: Challenges of diagnosis and treatment. Am Fam Physician . 2004;69(11):2609-2616.

14. Abdelkarim A, Levi DS, Tran B, Ghobrial J, Aboulhosn J. Fenestrated Transcatheter ASD Closure in Adults with Diastolic Dysfunction and/or Pulmonary Hypertension: Case Series and Review of the Literature. Congenit Heart Dis . 2016;11(6):663-671. 
15. Schneider HE, Jux C, Kriebel T, Paul T. Fate of a modified fenestration of atrial septal occluder device after transcatheter closure of atrial septal defects in elderly patients. J Interv Cardiol . 2011;24(5):485-490.

16. Akagi T. Current concept of transcatheter closure of atrial septal defect in adults. $J$ Cardiol . 2015;65(1):17-25.

17. Kuwata S, Vierecke J, Gloekler S, Maisano F, Meier B, Nietlispach F. Left atrial appendage closure for "primary primary" prevention during percutaneous closure of septal defects in patients with large atria but no atrial fibrillation. Cardiol J . 2018;25(2):179-187.

18. Asmarats L, Cruz-González I, Nombela-Franco L, et al. Recurrence of Device-Related Thrombus after Percutaneous Left Atrial Appendage Closure. Circulation . 2019;140(17):1441-1443.

19. Dukkipati SR, Kar S, Holmes DR, et al. Device-related thrombus after left atrial appendage closure: Incidence, predictors, and outcomes.Circulation . 2018;138(9):874-885.

\section{FIGURE LEGEND}

Figure 1: Step-by-step illustration of left atrial appendage (LAA) and fenestrated atrial septal defect (ASD) closure. (A) Dilated atriums in patients with atrial septal defect and restrictive left ventricle; (B) Closure of the left atrial appendage under transesophageal echocardiography and fluoroscopy guidance; (C) Creation of fenestration at the device using the dilator of the delivery sheath; (D) Presence of fenestration at the ASD occluder; (E) 3D transesophageal echocardiogram looking from the right atrium showing the fenestration (arrow) on the septal occluder; and (F) left to right shunting across the fenestrated septal occluder on Color Doppler.

\section{TABLES}

Table 1. Baseline characteristics of the patients prior to the occlusion of the left atrial appendage.

Table 2. Short and long term procedural outcome

Figure 1:

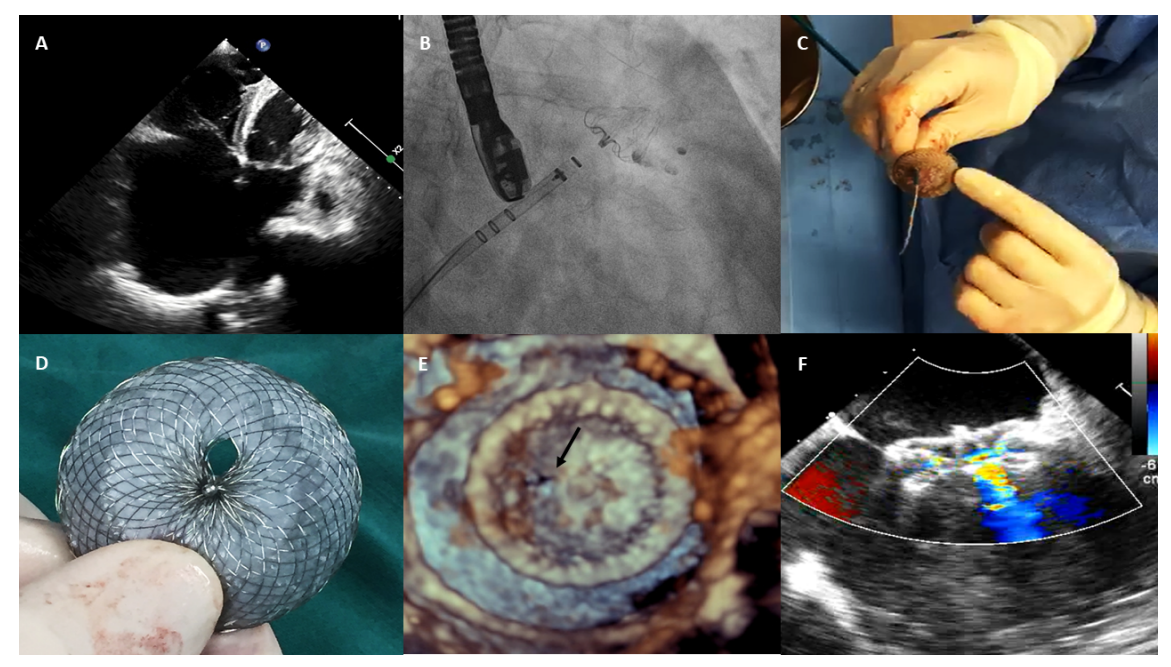

